\title{
Engineering Irrigation Drippers with Rechargeable N-halamine Nanoparticles for Antifouling Applications
}

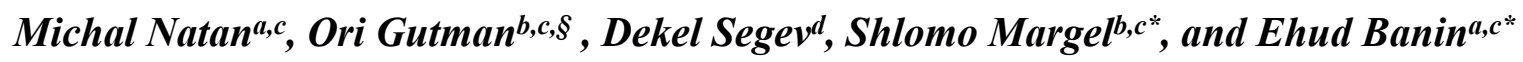

aThe Mina and Everard Goodman Faculty of Life Sciences, Bar-Ilan University, Ramat-Gan 52900, Israel. 'bThe Department of Chemistry, Bar-Ilan University, Ramat-Gan 52900, Israel. cThe Institute for Advanced Materials and Nanotechnology, Bar-Ilan University, Ramat-Gan 52900, Israel. dNetafim Ltd. kibbutz Magal 334500, Israel.

§Present address: The National drugs analysis lab, Israel police, Jerusalem, Israel

*Address corresponds to: ehud.banin@,biu.ac.il, shlomo.margel@mail.biu.ac.il 


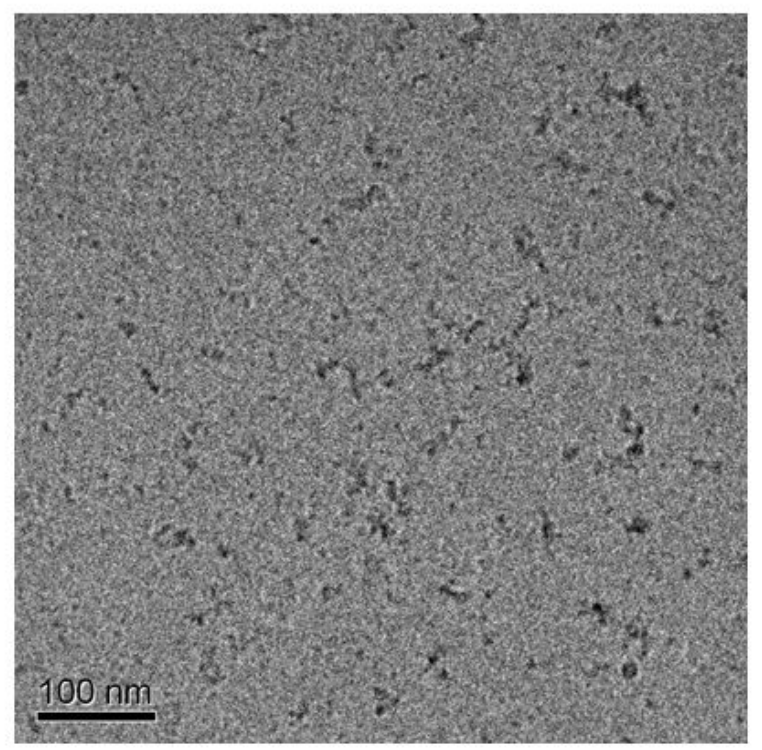

Figure S1. Cryo-TEM image of the P(MAA-MBAA)-CI NPs. The sample was prepared as described in the experimental section and observed in the Cryo-TEM.

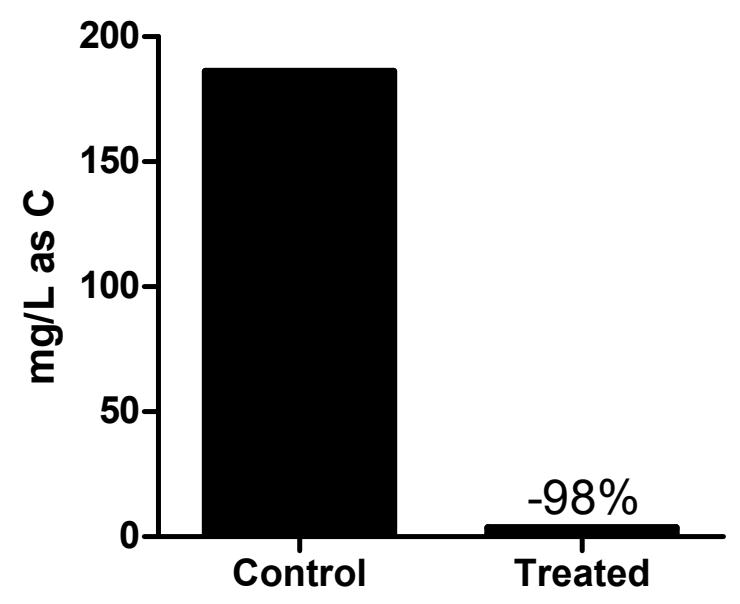


Figure S2. Chlorinated drippers prevent fouling. TOC analysis of the total biomass found on the chlorinated drippers versus the negative control, i.e. non-chlorinated drippers following 1 month incubation in Hazerimm, Israel. 